\title{
Lenvatinib, a molecule with versatile application: from preclinical evidence to future development in anti-cancer treatment
}

This article was published in the following Dove Press journal:

Cancer Management and Research

\author{
Monica Capozzi' \\ Chiara De Divitiis ${ }^{2}$ \\ Alessandro Ottaiano ${ }^{3}$ \\ Claudia von $\mathrm{Arx}^{4}$ \\ Stefania Scala ${ }^{5}$ \\ Fabiana Tatangelo 6 \\ Paolo Delrio' \\ Salvatore Tafuto'
}

'Department of Abdominal Oncology, Istituto Nazionale Tumori, IRCCS Fondazione "G. Pascale", Napoli, Italia; ${ }^{2}$ UOSD Oncology- AOU "San Giovanni di Dio e Ruggi D’Aragona", Salerno, Italia; ${ }^{3}$ SSD Innovative Therapies for Abdominal Metastases - Department of Abdominal Oncology, Istituto Nazionale Tumori, IRCCS - Fondazione "G. Pascale", Napoli, Italia; ${ }^{4}$ Department of Surgery and Cancer, Imperial College London, London, UK; ${ }^{5}$ Molecular Immunology and Immunoregulation, Istituto Nazionale Tumori, IRCCS - Fondazione "G. Pascale", Napoli, Italia; ${ }^{6}$ Department of Pathology, Istituto Nazionale Tumori, IRCCS - Fondazione "G. Pascale", Napoli, Italia

On behalf of ENETs (European NeuroEndocrine Tumor Society) Center of Excellence of Naples, Italy
Correspondence: Salvatore Tafuto Department of Abdominal Oncology, Istituto Nazionale Tumori di Napoli

"G. Pascale" IRCCS, National Cancer Institute, via M. Semmola, Naples 80131 , Italy

Tel +3908I 5903680

Email s.tafuto@istitutotumori.na.it

\begin{abstract}
Lenvatinib is an emerging multi-kinase inhibitor with a preferential antiangiogenic activity, which has shown efficacy in the treatment of renal cell carcinoma, differentiated thyroid cancer and hepatocellular carcinoma. It inhibits vascular endothelial growth factor receptor family (VEGFR1-3), fibroblast growth factor receptor family (FGFR1-4), platelet-derived growth factor receptor-alpha (PDGFR $\alpha$ ), tyrosine-kinase receptor (KIT) and rearranged during transfection receptor (RET). In this review we have evaluated the development from bench to bedside of lenvatinib. PubMed, MEDLINE and clinicaltrials.gov are the sources of data. Furthermore, the preclinical in vitro and in vivo data, as well as efficacy and toxicity results of lenvatinib in the clinic, are presented and discussed. Treatment with lenvatinib causes side effects (hypertension, proteinuria, fatigue and diarrhea), which are predominantly related to the inhibition of angiogenesis. For these reasons, the identification of biomarkers of efficacy and resistance to lenvatinib is a key challenge in order to select responsive patients. This review provides an overview on lenvatinib's clinical use, perspectives and indications for future development.
\end{abstract}

Keywords: angiogenesis, kinase inhibitors, microvessel density, tumors

\section{Plain language summary}

Lenvatinib inhibits many proteins involved in the formation of new vessels inside and outside tumors. This property has been elucidated by many researchers in a large number of experiments with cell lines and animals.

- Lenvatinib has anti-tumor clinical activity in patients affected by differentiated thyroid carcinoma, renal cancer and hepatocellular carcinoma.

- Direct and indirect evidences have suggested that lenvatinib can modulate tumor microenvironment and anti-tumor responses and that it could synergize with immune checkpoint blockade.

- To overcome or delay the resistance to lenvatinib anti-tumor effects, association of multiple drugs leading to the synchronous inhibition of different angiogenic pathways may be a future strategy.

- There are no biomarkers predicting response to lenvatinib, thus further studies are required to identify them. Another interesting and challenging issue will be the study of lenvatinib in combination with other drugs, and as sequential treatment after other lines of therapy including immunotherapies. 
There are no biomarkers predicting response to lenvatinib, thus further studies are required to identify them. Another interesting and challenging issue will be the study of lenvatinib in combination with other drugs, and as sequential treatment after other lines of therapy.

\section{Introduction}

In the past few years, advances in research and improved understanding of specific biological pathways involved in cancer progression have led to traditional chemotherapy being overtaken by the use of a new category of drugs designed to target specific proteins involved in the cells' proliferation and spread. ${ }^{1}$ Among these emerging treatments, the multi-kinase inhibitors are playing an increasingly important role as pleiotropic anti-tumor agents. Lenvatinib belongs to a class of small molecules that are particularly effective in counteracting tumor progression, by preventing phosphorylation and subsequent activation of many tyrosine kinases taking part in tumor cell proliferation and neo-angiogenesis. ${ }^{2}$ Lenvatinib has shown a versatile activity in different tumors, and has been demonstrated to be a valid therapeutic option in several neoplasms, with an easily manageable toxicity profile. However, no biomarkers are known to predict the response or resistance to it.

In this review we have evaluated the development from bench to bedside of lenvatinib. PubMed, MEDLINE and clinicaltrials.gov are the sources of data. Preclinical and clinical studies were selected using lenvatinib as the keyword.

\section{Pharmacokinetic and pharmacodynamic profile}

Lenvatinib is an antineoplastic compound belonging to the class of quinoline carboxiamides, corresponding to the chemical name of 4-[3-chloro-4-(cyclopropylcarbamoylamino) phenoxy]-7-methoxyquinoline-6-carboxamide in accordance with IUPAC nomenclature. ${ }^{3}$ Lenvatinib is rapidly well absorbed after oral administration, with $\mathrm{t}_{\max }$ from 1 to 4 hours. ${ }^{4}$ Data obtained from mass-balance studies fix the bioavailability at about $85 \%$. In vitro lenvatinib was extensively bound to human plasma proteins (98-99\%), predominantly to albumin, and less to $\alpha 1$-acid glycoprotein and $\gamma$-globulin. In humans, the median apparent volume of distribution of the first dose is between 50.5 and $92 \mathrm{~L}$, with a drug dose range from 3.2 to $32 \mathrm{mg}$. It is extensively metabolized by the liver, and eliminated prevalently in the feces. Plasma concentrations of the drug decline in bi-exponentially after $\mathrm{C}_{\max }$, and the half-life of drug is roughly 28 hours. ${ }^{5}$

Lenvatinib is an anti-tumor drug with a predominant anti-angiogenic effect. The mechanism of action of lenvatinib makes it a suitable treatment for different neoplasms that share the same biological patterns. It acts as a multityrosine kinase inhibitor and inhibits vascular endothelial growth factor receptor family (VEGFR1-3), fibroblast growth factor receptor family (FGFR1-4), plateletderived growth factor receptor-alpha (PDGFR $\alpha$ ), tyrosinekinase receptor (KIT) and rearranged during transfection receptor (RET), arresting neo-vessel assembly and maturation, and decreasing the vascular permeability of the tumor microenvironment. ${ }^{6}$ The kinase-inhibition profile of the drug has been established through in vitro cellular assays, measuring the half-maximal inhibitory concentration $\left(\mathrm{IC}_{50}\right){ }^{7,8}$ The $\mathrm{IC}_{50}$ values were calculated by ELISA and off-chip mobility shift assay (MSA) methods using a drug concentration rage of $0.3-10,000 \mathrm{nmol} / \mathrm{L}$. Lenvatinib showed a potent multi-kinase inhibition activity in a competitive manner: the most sensitive were VEGFR13 -associated kinases, with values of $\mathrm{IC}_{50} 4.7 \mathrm{nmol} / \mathrm{L}, 3.0$ $\mathrm{nmol} / \mathrm{L}$ and $2.3 \mathrm{nmol} / \mathrm{L}$, respectively. The others angiogenic factors shown to be sensitive to lenvatinib action are: RET, with an $\mathrm{IC}_{50}$ of $6.4 \mathrm{nmol} / \mathrm{L}$, KIT with $\mathrm{IC}_{50} 85$ nmol/L, FGFR1-4 with $\mathrm{IC}_{50}$ values of $61,27,52$ and 43 $\mathrm{nmol} / \mathrm{L}$, respectively, and PDGFR- $\alpha$ with $\mathrm{IC}_{50}$ of 29 $\mathrm{nmol} / \mathrm{L}$. The inhibition constants (Ki) were determined by a Dixon plot diagram using concentrations of lenvatinib in the range $0.3-260 \mathrm{nmol} / \mathrm{L}$ and six different concentrations of ATP. Lenvatinib inhibits the VEGFR family with a $\mathrm{Ki}$ of $1 \mathrm{nmol} / \mathrm{L}$, RET with a $\mathrm{Ki}$ of $1.5 \mathrm{nmol} / \mathrm{L}$, FGFR 1 with a Ki of $221 \mathrm{nmol} / \mathrm{L}$, FGFR2 with a Ki of $8.21 \mathrm{nmol} / \mathrm{L}$, and FGFR3 and KIT with Ki values of $151 \mathrm{nmol} / \mathrm{L}$ and 11 nmol/L, respectively. ${ }^{2-4}$

\section{Preclinical evidence: in vitro and in vivo experience}

The anti-tumor activity of lenvatinib has been studied using in vitro and in vivo models with different methods. The characteristics of these models are summarized in Table 1. Lenvatinib is effective in a broad panel of tumors. The anti-tumor activity, measured as tumor shrinkage, was strictly associated with the grade of tumor vascularization (vascular score). ${ }^{10}$ The pioneering work to assess the pharmacodynamic activity of lenvatinib was performed in 2008 by Matsui et al. ${ }^{8}$ The results showed that it 


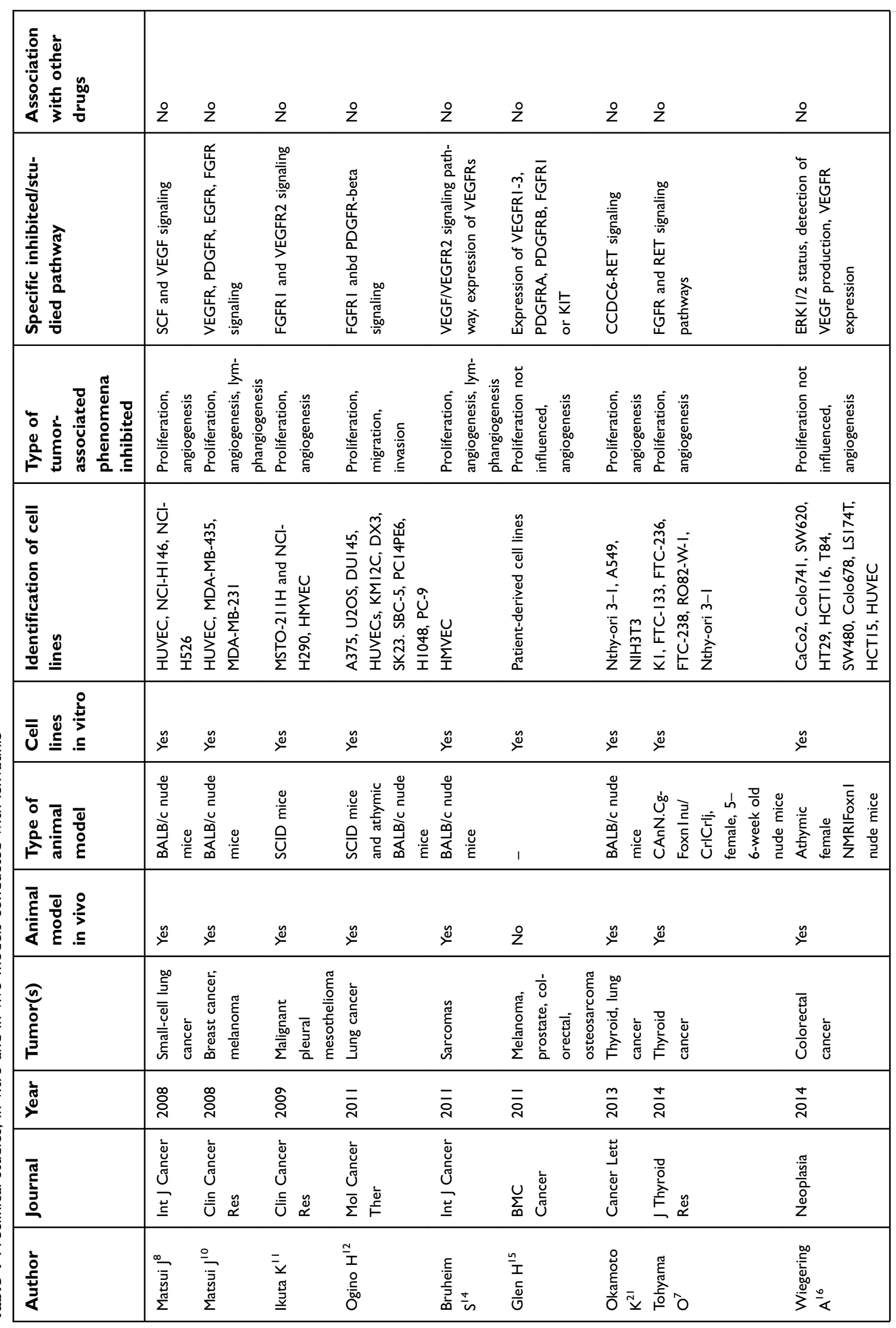




\begin{tabular}{|c|c|c|c|c|c|c|c|c|}
\hline 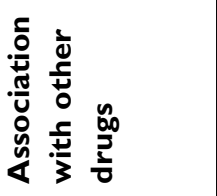 & 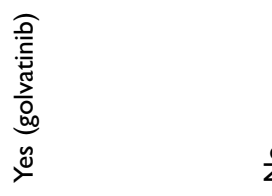 & zo & 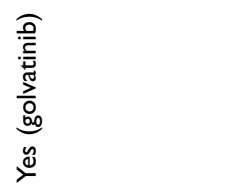 & zo & 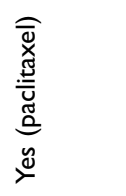 & zo & 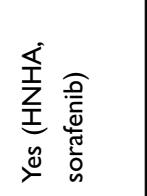 & 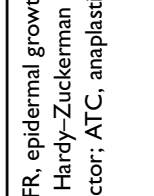 \\
\hline 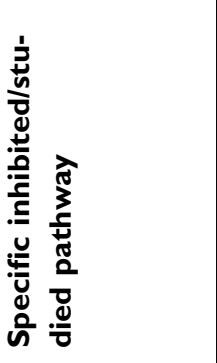 & 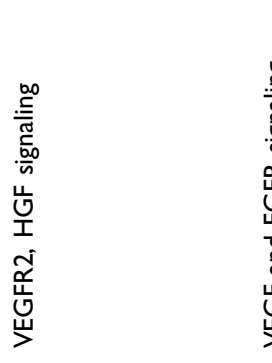 & 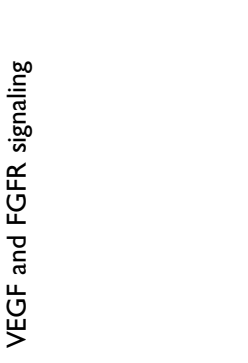 & 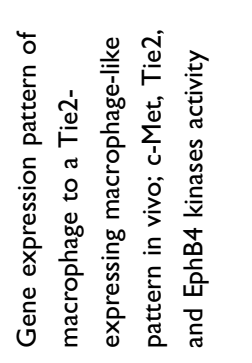 & 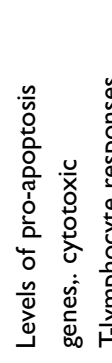 & : & 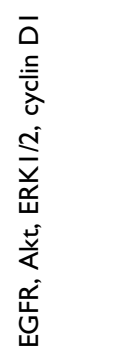 & 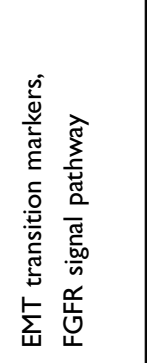 & 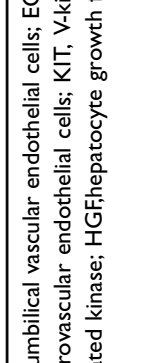 \\
\hline 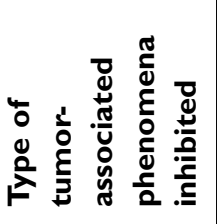 & 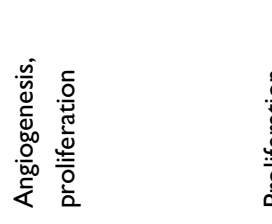 & 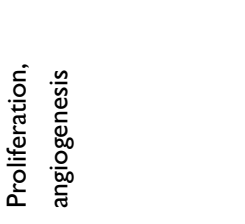 & 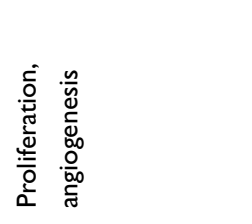 & 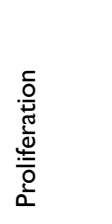 & \multicolumn{3}{|c|}{ 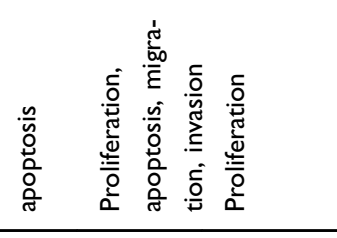 } & 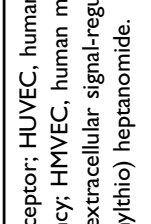 \\
\hline 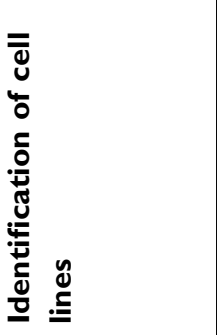 & 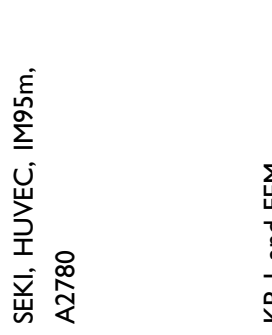 & 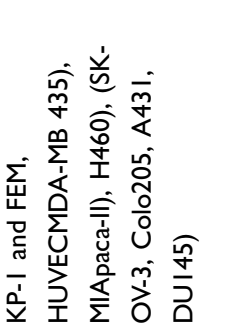 & 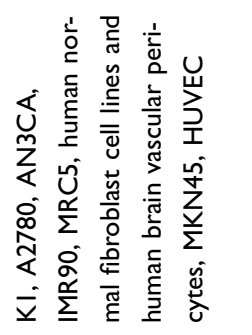 & 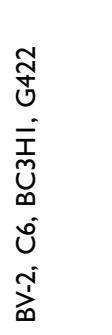 & 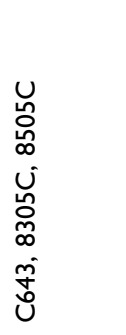 & 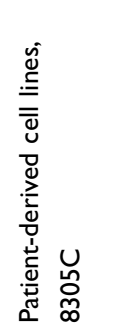 & 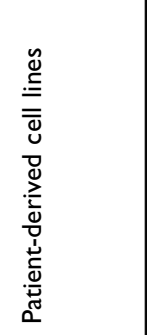 & 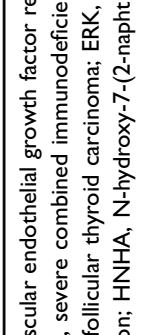 \\
\hline 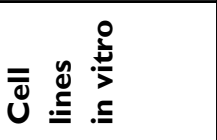 & $\stackrel{0}{\check{\nu}}$ & 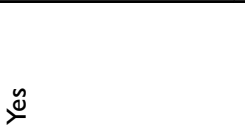 & $\stackrel{\mathscr{y}}{\check{\nu}}$ & zo & $\stackrel{\tilde{z}}{\nu}$ & $\stackrel{\check{\nu}}{\check{\nu}}$ & $\stackrel{\check{\nu}}{\check{\nu}}$ & 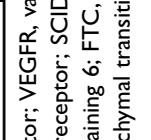 \\
\hline 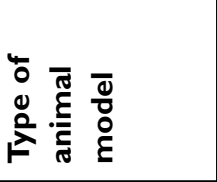 & 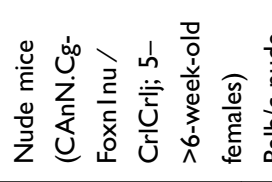 & 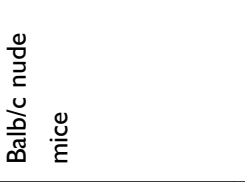 & 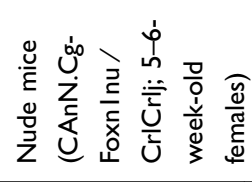 & 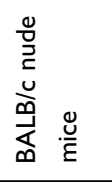 & 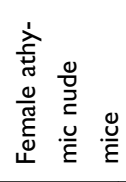 & 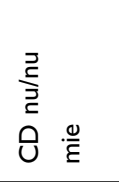 & 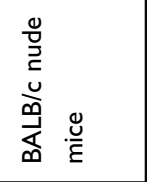 & 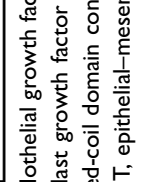 \\
\hline 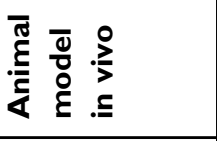 & $\stackrel{\check{\varpi}}{\check{\nu}}$ & ટ્ઁ & $\stackrel{\check{\nu}}{\check{\nu}}$ & 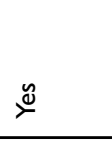 & $\stackrel{\check{\Perp}}{\check{n}}$ & $\check{\check{N}}^{\tilde{u}}$ & $\stackrel{\check{\nu}}{\check{\nu}}$ & 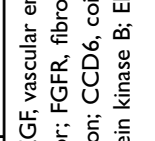 \\
\hline 言 & 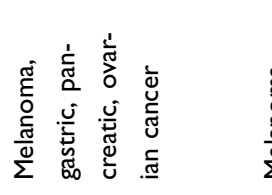 & 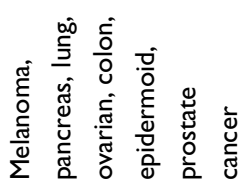 & 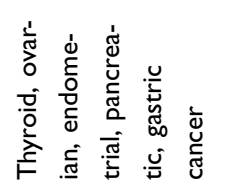 & 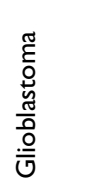 & 宸 & 岁 & 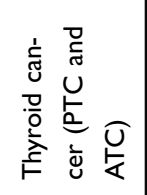 & 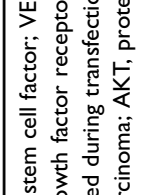 \\
\hline ঠ் & $\frac{\nabla}{\partial}$ & $\frac{\pi}{\circ}$ & $\frac{n}{2}$ & $\overline{\bar{i}}$ & $\overline{\bar{i}}$ & $\frac{\infty}{2}$ & $\frac{\infty}{i}$ & $\begin{array}{l}u \\
\dot{u} \\
\dot{u}\end{array}$ \\
\hline 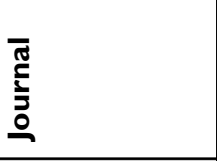 & 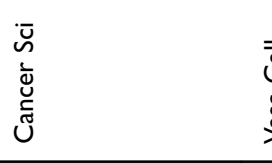 & $\begin{array}{l}\overline{\bar{U}} \\
\breve{y} \\
\nu\end{array}$ & 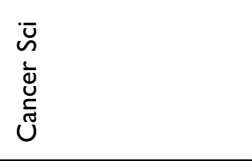 & 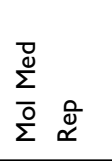 & छ芯 & 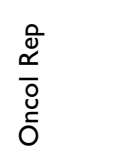 & $\begin{array}{l}\frac{\pi}{y} \\
\frac{\pi}{0} \\
\frac{0}{0} \\
z\end{array}$ & 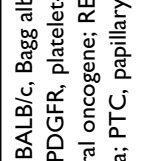 \\
\hline 高 & 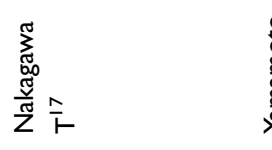 & 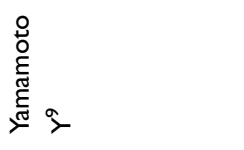 & $\begin{array}{l}\frac{\pi}{\sqrt[N]{ }} \\
\frac{\sqrt{\pi}}{2} \frac{\infty}{\succ}\end{array}$ & $\stackrel{\sigma}{\Xi}$ & $\begin{array}{l}\underset{\widetilde{U}}{\infty} \\
\stackrel{\infty}{\underline{\Xi}}\end{array}$ & 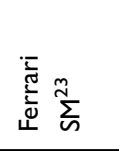 & 勇 & 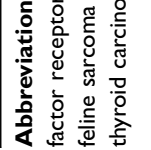 \\
\hline
\end{tabular}


inhibited in vitro both stem cell factor (SCF) and VEGFinduced tube formation in human umbilical vascular endothelial cells (HUVEC) in a dose-dependent manner, and also the phosphorylation of KIT and kinase insert domain receptor (KDR) in small-cell lung cancer cells. Furthermore, lenvatinib reduced tumor growth in mice through inhibition of KIT and VEGFR signaling. Similar results were obtained in breast cancer and melanoma, where lenvatinib was able to reduce proliferation, angiogenesis and lymphangiogenesis both in vitro and in vivo through VEGFR2 and VEGFR3 inhibition. In particular, a strong reduction of microvessel density was registered in both the primary tumor and metastatic nodules in xenograft models. ${ }^{10}$

Angiogenesis is a fundamental process for spreading of several tumors, such as malignant pleural mesotheliomas. These heterogeneous neoplasms often produce malignant pleural effusion and they are usually highly refractory to standard chemotherapy and radiotherapy. In 2009 a preclinical study with the purpose of investigating the activity of lenvatinib in these malignancies was realized by Ikuta et al, ${ }^{11}$ in which it was tested on three cell lines bearing different proangiogenic cytokine profiles; the results showed a reduction of cancer progression and a decrease in the number of proangiogenic factors. The final data confirmed the earlier outcome: in SCID xenograft mice, lenvatinib, in continuous administration, enhanced the survival in all three models through inhibition of FGFR1 and VEGFR2 signaling.

A subsequent study was conducted to investigate the therapeutic effect of lenvatinib in three lines of EGFR wild-type lung cancer cells, and then in SCID mice: ${ }^{12}$ the drug showed good efficacy in the inhibition of angiogenic processes in vitro, and shrinkage of tumor metastases leading to a prolongation of survival in mice models. Considering that, despite lung cancer treatment having recently improved with the use of EGFR inhibitors in mutated populations, the prognosis in EGFR wild-type subjects is still poor, ${ }^{13}$ the results obtained in these preclinical experiments are encouraging.

The preclinical activity of lenvatinib was also evaluated in malignant sarcomas, in a study conducted in 2011: ${ }^{14}$ in vitro, it did not show an anti-tumor activity on four sarcoma cell lines; however, the tumor vasculature shrinkage was evident in xenograft models.

Glen et $\mathrm{al}^{15}$ were the first to conduct experiments on patient-derived cell lines form melanoma, prostate cancer, colorectal cancer and osteosarcoma. Similarly to
Wiegering et al, ${ }^{16}$ they demonstrated that lenvatinib did not affect directly tumor cell proliferation either in vitro or in vivo; instead, the drug delayed tumor growth by reducing capillary density and tumor volume in treated mice compared to the control group. ${ }^{16,17}$ Inhibition of VEGFR signaling was in any case crucial to obtain this effect. New biological drugs can represent an important resource, particularly for those malignancies with limited treatment options; preclinical studies published in recent years have extended the study of the anti-neoplastic properties of lenvatinib in gastric, pancreatic, ovarian, glioblastoma and endometrial cancers. ${ }^{9,17-19}$ Studied cell lines and methods are summarized in Table 1.

Lenvatinib has been demonstrated to be particularly effective in thyroid cancer. In February 2015, it received approval from the US Food and Drug Administration (FDA) for the treatment of differentiated thyroid cancer (DTC). Thyroid cancer can be classified according to histological derivation and grade of differentiation: ${ }^{20}$ the most common forms are papillary (PTC) and follicular (FTC) thyroid carcinomas, which are differentiated types and account for more than $80 \%$ of thyroid tumors. Hurthle cell thyroid cancer is classified as a type of FTC and it accounts for about $5 \%$ of all thyroid cancers. Medullary thyroid carcinoma (MTC) develops from $\mathrm{C}$ cells in the parafollicular regions of the gland; it is more aggressive and less differentiated than PTC and FTC, and occurs in less than $10 \%$ of cases. Anaplastic thyroid carcinoma (ATC) is the most undifferentiated and aggressive form, with poor prognosis; it occurs in about $2 \%$ of cases. The description of the clinical behavior of thyroid cancers is beyond the scope of the present review; however, differentiated forms generally occur with more localized, less aggressive and better prognosis disease compared to ATC. Many preclinical studies have demonstrated that DTC cells are particularly sensitive to lenvatinib-induced inhibition of proliferation and angiogenesis in both in vitro and in vivo models. ${ }^{7,18,21-23}$ Pathways significantly inhibited in DTC cell lines are FGFR, CCDC6-RET, Met, Tie2 and EphB4 signaling. Lenvatinib was able to reduce proliferation and tumor vascularization in nude mice xenografts derived from DTC, MTC and ATC lines. Although DTC represents the first approved indication for the use of lenvatinib in the clinic, further preclinical and clinical evaluations are warranted for ATC, the rare form of undifferentiated thyroid cancer with a high mortality rate. In fact, ATC cells (both established and patient-derived cell lines) are also sensitive to lenvatinib inhibition of 
proliferation in both in vitro and in vivo models, and the drug has shown to be effective alone ${ }^{23}$ and in combination with paclitaxel. ${ }^{22}$ Lenvatinib is currently being investigated in the USA in a phase II study in ATC (NCT02657369).

\section{Modulation of immune cells and tumor microenvironment by lenvatinib}

In recent years, direct and indirect evidence has suggested that lenvatinib can modulate the tumor microenvironment and anti-tumor responses; however, the topic is largely unexplored. Inhibition of VEGFR-1 signaling has some pleiotropic effects on the tumor microenvironment, including induction of hypoxia and modulation of tumorassociated macrophages (TAMs). ${ }^{24}$ The first phenomenon is associated with an increased expression of programmed death-ligand 1 (PD-L1); the second consists of promoting the TAM-M1 phenotype, which is positively involved in anti-tumor immunity (recruitment and activation of $\mathrm{T}$ cells). Thus, lenvatinib could synergize with immune checkpoint blockade by promoting TAMs' antitumor M1 phenotype in the tumor microenvironment. ${ }^{24,25}$ In addition, PIGF and VEGF-A are involved through VEGFR-1 in mobilizing hematopoietic progenitor cells from the bone marrow; ${ }^{26}$ these cells are directly able to stimulate growth and vascularization of the primary neoplasm. In fact, they promote the TAM-M2 phenotype (favoring the immunological escape) and attract circulating cancer cells to the metastatic sites. ${ }^{27}$ Studies in human and mouse models of the ATC microenvironment have shown high PDL1 expression and a high frequency of $\mathrm{T}$ cells. ${ }^{28}$ This fact has prompted investigations on immunotherapy in ATC. However, clinical data in 22 patients enrolled in the phase Ib Keynote-028 study demonstrated that single-agent immunotherapy with pembrolizumab has limited clinical activity, with a response rate of $9.1 \%$ and a 6-month progression-free survival (PFS) rate of $58.7 \%{ }^{29}$ Since TAMs represent a high proportion of ATC and DTC volume, the association of immunotherapy with lenvatinib could provide a system to improve clinical results by taking advantage of the positive impact of lenvatinib on the tumor microenvironment. In addition, regulatory $\mathrm{T}$ cells express VEGFR-2, and stimulation through VEGF-A is connected with PD-1 increase and inhibition of $\mathrm{T}$ cells. ${ }^{30} \mathrm{~A}$ preclinical study in renal cell carcinoma (RCC) demonstrated that cellular immunotherapy in association with lenvatinib significantly increases survival in murine models; ${ }^{31}$ furthermore, the association of lenvatinib and pembrolizumab has shown efficacy in a phase I study, particularly in RCC and melanoma, ${ }^{32}$ with a high disease-control rate.

\section{Results from phase I trials}

Several phase I trials have been conducted with the purpose of exploring the efficacy and safety profile of levatinib. Most of the clinical studies were designed with the classic $3+3$ dose-escalation scheme. A schematic summary of these trials is provided in Table 2 .

To test the preliminary evidence of drug efficacy in humans, an open-label, phase I, single-center, doseescalation trial was conducted with the purpose of establishing the maximum tolerated dose (MDT) and the dose-limiting toxicity (DLT) for lenvatinib in patients with advanced solid tumors. ${ }^{33}$ Circulating endothelial cells (CECs) and circulating endothelial progenitor cells (CEPs) are associated with vascular turnover into tumor areas; thus, quantification of these cells may provide a biomarker of tumor neoangiogenesis. ${ }^{34,35}$ In the present study, plasma angiogenic proteins using a bioplex multiple assay, and CECs and CEPs through flow cytometry were measured before lenvatinib administration and at day 15 in cycle 1 in order to investigate possible correlations between drug efficacy and specific biomarkers. In addition, CEC and CEP were divided into two subgroups: c-kit positive $(+)$ and c-kit negative (-). Lenvatinib reduced the subgroups of CEPs and CECs that express c-kit, probably through the inhibition of c-kit kinase in bone marrow.

The administration schedule was started from $0.5 \mathrm{mg}$ per os twice daily (BID) in a regimen of 2 weeks on/1 week off treatment. Overall, 27 patients were enrolled, in nine cohorts ranging from $0.5 \mathrm{mg}$ to $20 \mathrm{mg}$ per os BID; in five subjects serious adverse events occurred, possibly related to the study drug: hypertension, hemorrhage, pneumonia, dyspnea and decreased platelet count. DLT included grade 3 AST/ALT at $16 \mathrm{mg} \mathrm{BID}$ and grade 3 platelet count at $20 \mathrm{mg}$ BID. The MTD was reached at $13 \mathrm{mg}$ BID. One patient reached a partial response (PR), and stable disease (SD) was documented in 21 patients (84\%). The reduction in c-kit(+) CECs was associated with lenvatinib treatment duration while, among soluble factors, only pre-dose levels of stromal derived factor 1 alfa (SDF1alfa) showed a significant inverse correlation with lenvatinib treatment duration.

In another non-randomized, open-label, dose-escalation phase I trial, Boss et $\mathrm{al}^{36}$ evaluated the MTD safety and 


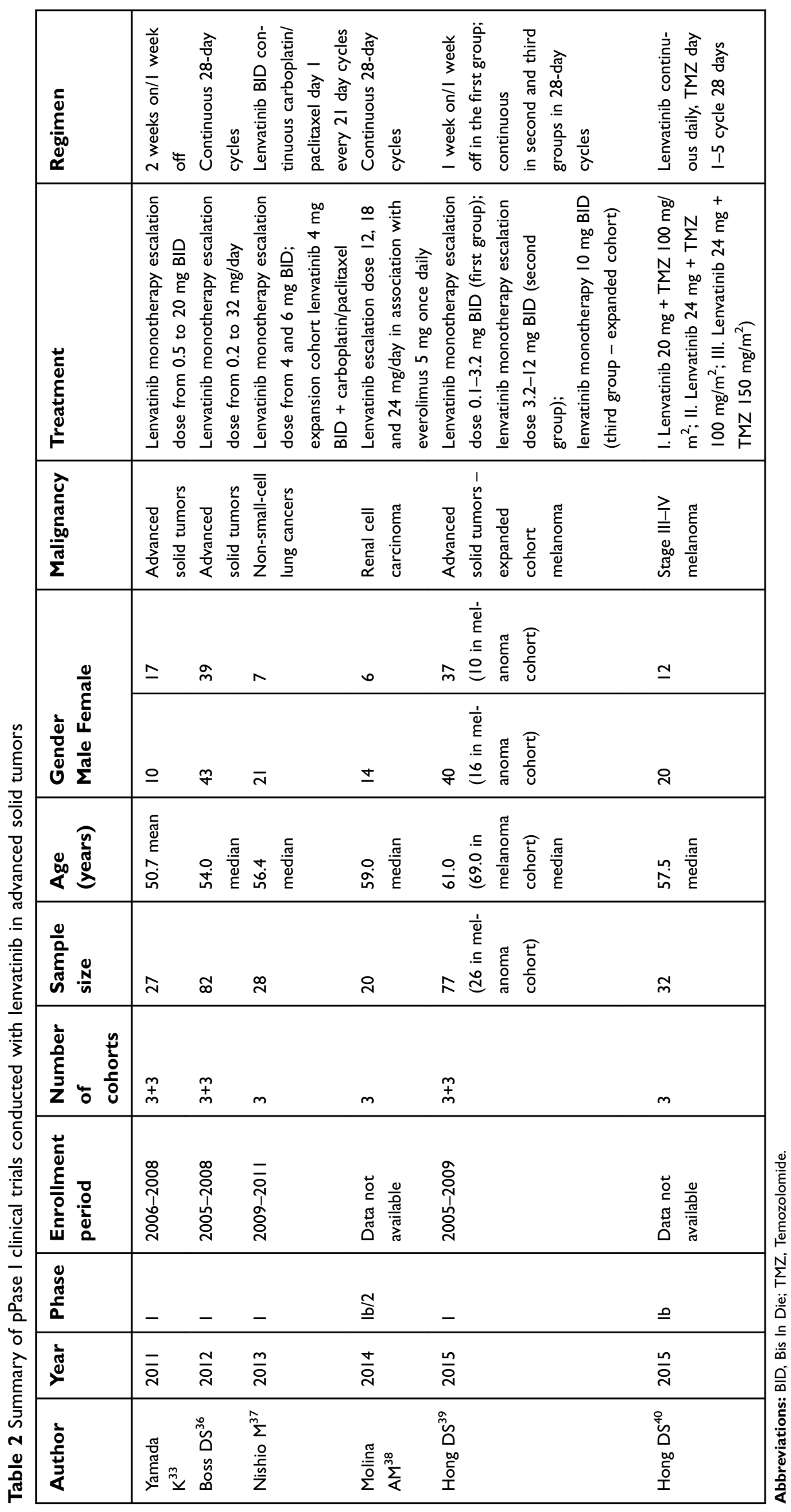


efficacy of lenvatinib in 82 patients with advanced refractory solid tumors. The schedule consisted of once-daily oral administration on a 28-day continuous cycle until unacceptable toxicity or disease progression. The patients received a starting dose of $0.2 \mathrm{mg}$, increasing by $100 \%$ in subsequent cohorts until any grade 2 toxicity occurred, after which the dose increases were 50\% until MTD was determined. The most frequently adverse events were diarrhea (45\%), hypertension $(40 \%)$ and nausea $(37 \%)$ as cumulative treatment toxicity. The DLT was $32 \mathrm{mg} / \mathrm{day}$, at which grade 3 proteinuria occurred, and the MDT was established at $25 \mathrm{mg} /$ day. Clinical benefit was observed in $55 \%$ of the population, seven patients had a PR and 38 had SD as the best result.

Lenvatinib was also evaluated in combination therapy with carboplatin and paclitaxel in patients with non-smallcell lung cancer, in a phase I, open-label, dose-finding study. ${ }^{37}$ In this trial, 28 patients were enrolled and received oral lenvatinib BID and paclitaxel/carboplatin intravenously on day 1 , every 21 days. The MTD observed was $4 \mathrm{mg}$ BID for lenvatinib. Among 22 patients treated with $4 \mathrm{mg}$ BID, 14 (64\%) had a PR and 1 (5\%) a complete response $(\mathrm{CR})$. Common toxicities included thrombocytopenia, occurring in $100 \%$ of subjects, neutropenia and leucopenia in $95 \%$, proteinuria in $77 \%$ and hypertension in $73 \%$.

A phase Ib, multicenter, open-label $3+3$ doseescalation study was performed to investigate the safety, MTD and efficacy of lenvatinib in association with everolimus in patients with advanced and metastatic RCC. ${ }^{38}$ Patients were treated in a continuous regimen of administration with an initial dose of lenvatinib $12 \mathrm{mg}$ and everolimus $5 \mathrm{mg}$ once daily. The escalation doses of the drug were $18 \mathrm{mg}$ and $24 \mathrm{mg}$ in subsequent cohorts. Everolimus was always administered at a dose of $5 \mathrm{mg}$ once daily. The objective responses were PR in $30 \%$ of all patients, SD in $50 \%$ and durable SD ( $\geq 23$ weeks) in $20 \%$ of them. In 2015 , Hong et $\mathrm{al}^{39}$ realized a phase I, open-label, modified 3+3 model study design to explore the efficacy, safety and biological activity of lenvatinib in patients with advanced solid tumors and in an expanded cohort of melanoma patients. The drug was administered per os according to three schedules: the first one was an escalation dose ranging from $0.1 \mathrm{mg}$ to $3.2 \mathrm{mg}$ administered $\mathrm{BID}$ in a regimen of 1 week on/1 week off; the second one was an escalation dose ranging from $3.2 \mathrm{mg}$ to $12 \mathrm{mg}$ administered BID in a continuous regimen; the third was $10 \mathrm{mg}$ BID. Seventy-seven patients were enrolled, 26 in the expanded melanoma cohort. The MTD was established in the $10 \mathrm{mg}$ BID group. Among them, nine patients presented a PR (11.7\%), 40 had a SD (51.9\%), response was not assessed in 10 patients; three patients had a PR and seven had a durable SD in the melanoma cohort. The most common adverse events were hypertension (43\%), fatigue (42\%) and proteinuria (39\%).

Furthermore, Hong's research group evaluated lenvatinib in patients affected by advanced melanoma in combination with temozolomide. ${ }^{40}$ In this trial, 32 subjects were enrolled at three dose levels (lenvatinib $20 \mathrm{mg}+$ temozolomide $100 \mathrm{mg} / \mathrm{m}^{2}$, lenvatinib $24 \mathrm{mg}+$ temozolomide $100 \mathrm{mg} / \mathrm{m}^{2}$, and lenvatinib $24 \mathrm{mg}+$ temozolomide $150 \mathrm{mg} / \mathrm{m}^{2}$ ) in a schedule of continuous once-daily administration of lenvatinib and infusion of temozolomide on day 1-5 of 28-day cycles. The treatment was well tolerated and only one patient had a DLT, while MTD was not reached. Laboratory data suggested that the pharmacokinetics of lenvatinib was not influenced by the concomitant administration of temozolomide. However, of the overall population, 6 achieved a PR (18.8\%), and 15 a SD $(46.9 \%)$, much lower than results obtained with the ipilimumab/nivolumab combination $(61 \%$ objective response rate: 44 out of 72 total patients). ${ }^{41}$

The majority of the lenvatinib-related adverse events documented in these phase I studies, such as hypertension and proteinuria, were consistent with lenvatinib's activity in inhibiting VEGFR and angiogenesis. Some of them, for example hypertension, required pharmaceutical management with anti-hypertensive treatment or lenvatinib dose reductions.

\section{Results from phase II/III clinical trials \\ Renal cell carcinoma (RCC)}

Expression of VEGFR is one of the most common characteristics of RCC, which is currently treated with VEGF and mTOR inhibitors in monotherapy. The encouraging results obtained with lenvatinib in preclinical studies provided the rationale to investigate its effectiveness in clinical settings. In metastatic RCC, lenvatinib has been tested in a randomized phase II trial, as second line treatment in patients progressing after anti-VEGF first line treatment. ${ }^{42}$ The primary goal was PFS. Subjects were randomly assigned to receive everolimus alone, lenvatinib alone or a combination of them, in a ratio of $1: 1: 1$. Patients treated with lenvatinib alone had a PFS of 7.4 months compared to 5.5 months with everolimus alone (HR 0.61, 95\% CI 
$0.38-0.98, P=0.048)$. In addition, lenvatinib plus everolimus combination treatment improved PFS to 14.6 months compared with 5.5 months for everolimus alone (HR 0.40, $95 \%$ CI $0.24-0.68, P=0.0005$ ), but the combination agents results were not significantly improved with respect to lenvatinib as a single agent. Thus, lenvatinib improved PFS in patients with metastatic RCC who have progressed after one previous anti-VEGF first line therapy.

\section{Thyroid cancer}

The first supportive data on lenvatinib efficacy and safety in patients affected by advanced MTC were provided by Schlumberger et al, in a phase II, multicenter, open-label, single-arm clinical trial. ${ }^{43}$ Fifty-nine patients affected by advanced MTC were enrolled to receive lenvatinib at a dose of $24 \mathrm{mg}$ daily, in a schedule of 28-day cycles. The objective response rate (ORR) was 36\% (95\% CI 24-49\%) and the disease control rate (DCR; CR + PR + SD) was $80 \%$ (95\% CI 67-89\%). Among 51 patients tested, 13 had a decrease in C-terminal agrin fragment (CAF) levels following 8 days of treatment with lenvatinib, and this reduction was associated with clinical outcome.

Lenvatinib was tested in advanced, progressive, radioiodine-refractory DTC (RR-DTC) in a phase II trial conducted in $2015 .{ }^{44}$ In the study, 58 patients were treated with lenvatinib $24 \mathrm{mg} /$ day in 28-day cycles. The primary endpoint was ORR, while secondary endpoints included PFS and safety. The data showed an ORR of $50 \%$ (95\% CI $37-63 \%$ ) after 14 months of follow-up and a PFS of 12.6 months $(95 \%$ CI 9.9-16.1).

The positive results obtained in this phase II study prompted a randomized, double-blind phase III trial, in which lenvatinib was compared to placebo, in 261 patients affected by RR-DTC. ${ }^{45}$ The primary endpoint was PFS, and the secondary endpoints were overall survival (OS), ORR and safety. The median PFS in the treated group was 18.3 months, compared to 3.6 months in the placebo group (HR $0.21,99 \%$ CI $0.14-0.31, P<0.001$ ). The response rate was $64.8 \%$ (165 PR and four CR). The most important adverse events in the treated group included hypertension (67.8\% of patients), diarrhea (59.4\%) and asthenia (59\%).

\section{Hepatocellular carcinoma (HCC)}

A Phase II study was conducted in Japan and Korea during 2010 and 2011 to test lenvatinib in patients with advanced, unresectable HCC. ${ }^{46}$ In this study, it showed a good efficacy with an acceptable profile of toxicity; however, lenvatinib exposure was influenced by the patients weight, and an adjustment of drug dosage in the early phases of studied was required. The primary endpoint was time to progression (TTP), while secondary endpoints were ORR, DCR and OS. The TTP was 7.4 months $(95 \%$ CI 5.5-9.4); 17 patients had a PR and 19 had a SD (37\% ORR, 78\% DCR). Median OS was 18.7 months (95\% CI 12.7-25.1). The most common adverse event was hypertension $(76 \%)$.

Following these promising results, a phase III trial was performed to compare lenvatinib and sorafenib in first line treatment of patients with unresectable $\mathrm{HCC}$ and Child-Pugh A liver status. ${ }^{47}$ This was an open-label, multicenter trial: 954 patients, from 20 countries, were enrolled and randomly assigned to lenvatinib $(n=478)$ and sorafenib $(n=476)$. The results showed a non-inferior activity of lenvatinib compared to sorafenib: median OS was 13.6 months for lenvatinib and 12.3 months for sorafenib (HR $0.92,95 \%$ CI $0.79-1.06$ ). The most common drug-related adverse event was hypertension, which occurred in 201 patients (42\%) treated with lenvatinib, and palmar-plantar erythrodysaesthesia, occurring in 249 patients (52\%) treated with sorafenib. Characteristics of these clinical trials are summarized in Table 3.

\section{Role of lenvatinib in overcoming drug resistance and mechanisms of resistance to lenvatinib}

Resistance and recurrence are among the major challenges facing the treatment of tumors. According to recent findings, cancer stem cells are implicated in drug resistance and are the main factor responsible for the formation of distant metastases through an epithelial-mesenchymal transition (EMT) process. ${ }^{48}$ In tumor growth, tissue invasion is crucial for cancer progression, and most patient deaths are caused by distant metastases. The role of lenvatinib in the multi-resistant setting has been explored in a study conducted on patient-derived sorafenib-resistant poorly differentiated thyroid cancer cells, in which lenvatinib in association with the histone deacetylase inhibitor HNHA was demonstrated to block the EMT process through interference with the FGFR signaling pathway. ${ }^{49}$ Although the molecular mechanism involved in EMT regulation has not been fully elucidated, several studies focusing on EMT in stem cell models have pointed out that both p21 and p53 activation play a key role in the suppression of EMT. ${ }^{50}$ In patient-derived thyroid cancer cell lines, the combination of HNHA and lenvatinib demonstrated 


\begin{tabular}{|c|c|c|c|c|c|}
\hline $\begin{array}{l}\frac{\partial}{\frac{o}{a}} \\
\frac{\partial}{u} \\
\frac{\partial}{u}\end{array}$ & 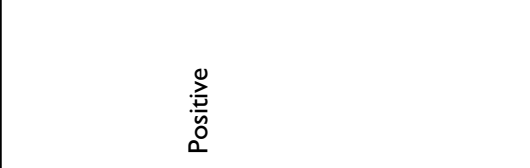 & $\begin{array}{l}\sum_{2}^{0} \\
\text { : } \\
0 \\
0\end{array}$ & 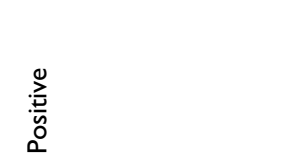 & : & $\sum_{\substack{\bar{y} \\
0}}^{0}$ \\
\hline 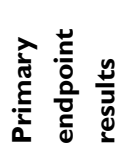 & 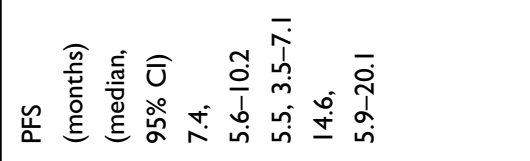 & 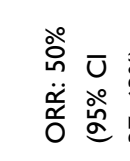 & 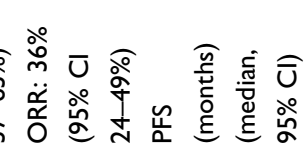 & 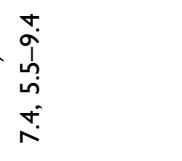 & 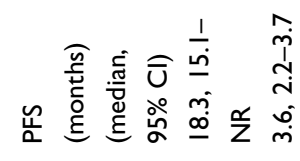 \\
\hline 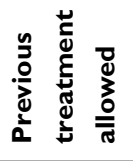 & 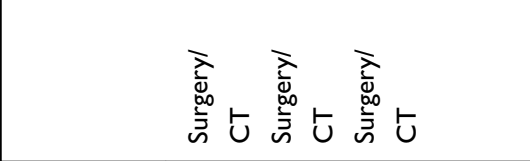 & $\longleftarrow$ & 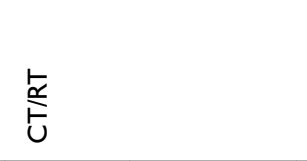 & 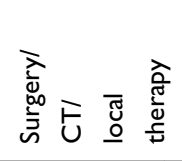 & 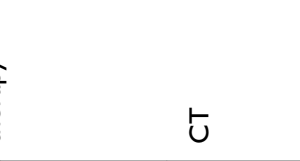 \\
\hline 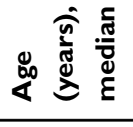 & กิธ & 3 & $\stackrel{0}{\dot{n}}$ & $\begin{array}{l}n \\
0 \\
0 \\
0\end{array}$ & \pm 5 \\
\hline \multirow{2}{*}{ 吾 } & $\underline{\underline{\simeq}} \underline{\underline{0}}$ & $\stackrel{\sim}{\sim}$ & ส & $\underline{m}$ & $\stackrel{\check{\Upsilon}}{\sim}$ 亡ீ \\
\hline & 同 $\stackrel{\infty}{m} \stackrel{n}{m}$ & ఫे & $\hat{m}$ & $\stackrel{m}{m}$ & $\stackrel{\Perp}{\simeq}$ \\
\hline 莺 & กู & $\stackrel{\infty}{n}$ & ติ & fo & $\bar{i} \overline{\underline{m}}$ \\
\hline 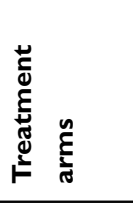 & \multicolumn{2}{|c|}{ 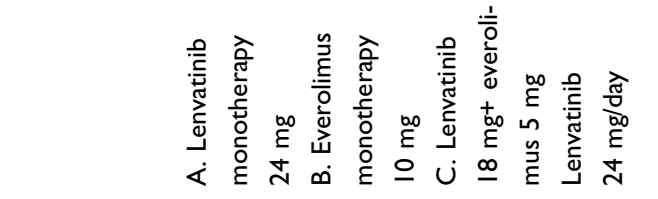 } & 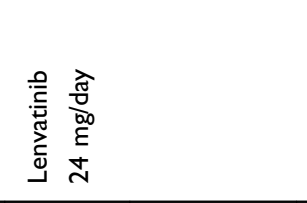 & 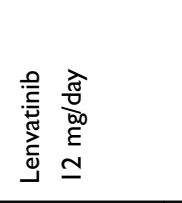 & 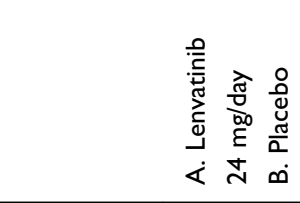 \\
\hline 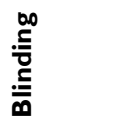 & 우 & 우 & 우 & 우 & $\stackrel{\check{\nu}}{\sim}$ \\
\hline 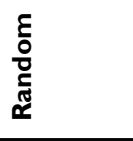 & $\stackrel{\check{\varpi}}{\tau}$ & ¿ & z & ㅇ & $\stackrel{y}{\sim}$ \\
\hline 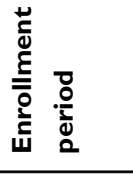 & $\begin{array}{l}\frac{m}{2} \\
\stackrel{\text { I }}{\grave{n}}\end{array}$ & 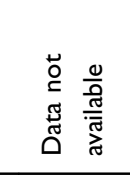 & 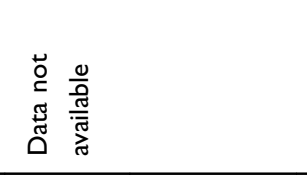 & $\begin{array}{l}\bar{o} \\
\bar{c} \\
\bar{d}\end{array}$ & $\frac{\bar{c}}{\frac{1}{2}}$ \\
\hline 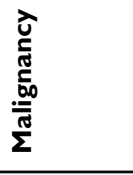 & $\underset{\sim}{\cup}$ & 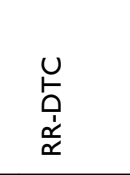 & $\underset{\Sigma}{U}$ & 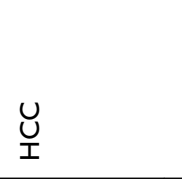 & $\begin{array}{l}u \\
\vdash \\
\stackrel{\alpha}{\alpha} \\
\dot{\alpha}\end{array}$ \\
\hline 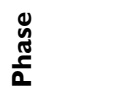 & $=$ & $=$ & $=$ & $=$ & $\equiv$ \\
\hline ঠัঠ & $\stackrel{\text { 음 }}{\frac{N}{2}}$ & $\stackrel{\text { 음 }}{\frac{N}{2}}$ & $\stackrel{\circ}{\bar{\alpha}}$ & $\frac{\circ}{2}$ & $\stackrel{\text { 음 }}{\circ}$ \\
\hline 高 & 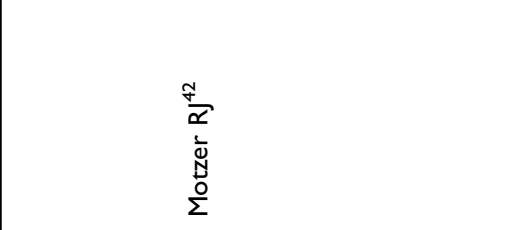 & 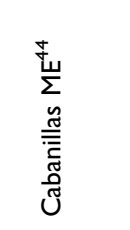 & 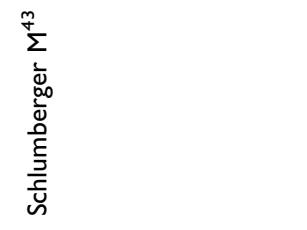 & 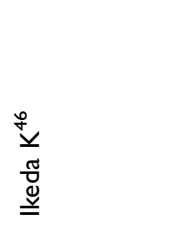 & 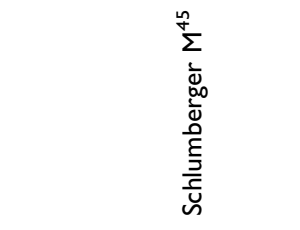 \\
\hline
\end{tabular}




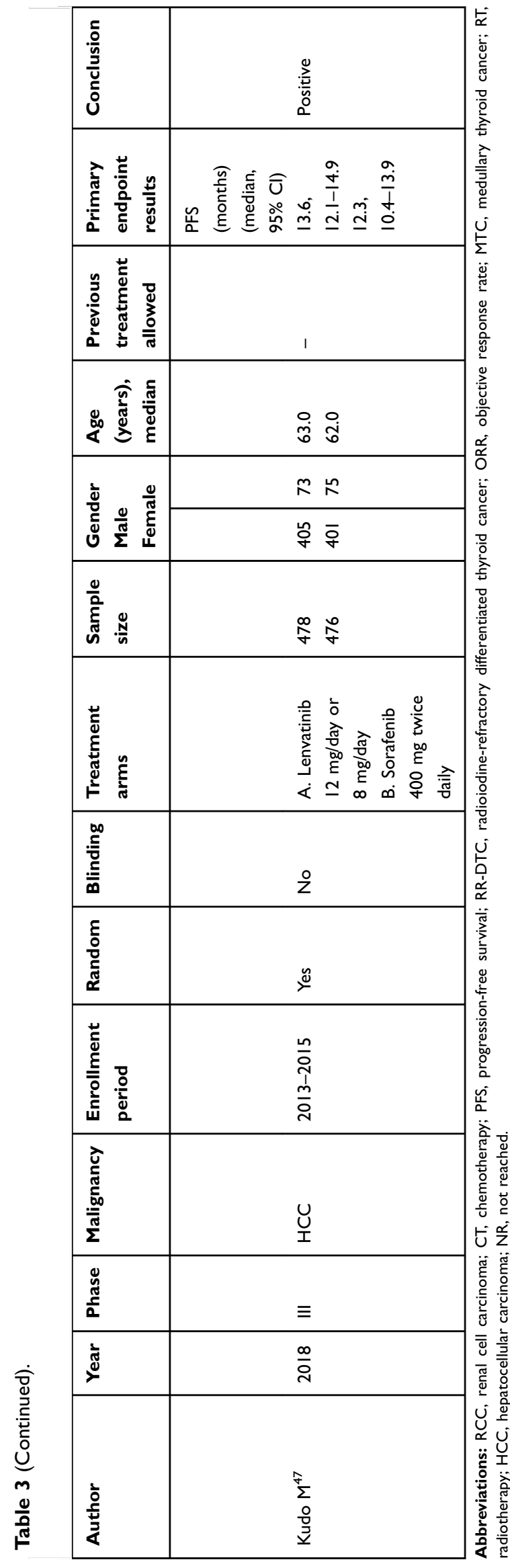

a synergistic anti-cancer activity, inducing apoptosis and cell-cycle arrest. Immunoblot analyses of proteins performed on cell lines showed increased levels of p21 and p53, and decreased levels of cyclin D1 and CDK4 compared to the group treated with HNHA and sorafenib. Lee et $\mathrm{al}^{49}$ provided evidence that lenvatinib reduced $\beta$-catenin nuclear localization; $\beta$-catenin is one of the main factors involved in the development of metastasis via EMT activation in cancer. Furthermore, the Ki67 proliferation marker expression was reduced in cells treated with lenvatinib compared to cells treated with HNHA and sorafenib. In NeuroEndocrine Tumors (NETs) the Ki67 levels in tissue samples is one of the most important approaches to grade the tumor and guide the treatment, thus the effect of lenvatinib on this biomarker is interesting and could translate into clinical applications (prognostic and/or therapeutic).

Furthermore, cancer cells could also become resistant to anti-angiogenic drugs through several mechanisms. ${ }^{51}$ In fact, despite the clinical results, particularly in HCC and DTC, most patients develop resistance to lenvatinib. There are no specific studies on the mechanisms of resistance to lenvatinib; however, two main mechanisms could be involved: activation of alternative pathways or upregulation of receptors on tumor cells. ${ }^{52,53}$ Among alternative pathways, preclinical studies have highlighted the role of c-MET, ${ }^{54}$ MEK, ${ }^{55}$ PI3K-AKT cascade ${ }^{56}$ and mTOR. ${ }^{57}$

Association of multiple drugs leading to the synchronous inhibition of different angiogenic pathways may be a strategy to overcome or delay resistance. One of the clinical translations of this hypothesis is the combination treatment of multi-target tyrosine kinases, such as the association of lenvatinib and golvatinib. This association has been tested in a few recent preclinical studies and demonstrated to be safe and to improve anti-tumor results. ${ }^{17,18}$

\section{Conclusion and future perspectives}

Although lenvatinib failed to demonstrate a strong antiproliferative function, its anti-tumor activity can be attributed to a reduction in microvessel density and a substantial shrinkage of tumor bulk.

We were prompted to share our experience in daily use of lenvatinib, taking advantage of participation in an international pivotal phase II clinical trial (TALENT) evaluating lenvatinib in metastatic G1/G2 enteropancreatic neuroendocrine tumors. The study is currently ongoing, the primary endpoint is ORR and the results are expected 
in 2019. Neuroendocrine neoplasms (NENs) are hypervascularised tumors and the application of lenvatinib on lowgrade NENs is based on overexpression in neuroendocrine cancer cells of proangiogenic molecules and receptors such as VEGF/VEGFR, FGF/FGFR, PDGF/PDGFR and EGF/EGFR. ${ }^{58,59}$ Adequate selection of patients is still an unmet need and to date there are no biomarkers to guide lenvatinib-based therapies. Researchers should attempt to fill this gap through high-profile collaborations, international databases, molecular and biological banks, and genome-wide profiling approaches. In addition, further benefits for patients will derive from a better understanding of the complete profile of toxicity as well as the best timing and schedule for lenvatinib administration. The mechanism of action of lenvatinib could account for versatile applications of the drug in cancer treatment; however, its clinical use is based predominantly on phase I/II studies. Phase III clinical trials exploring lenvatinib efficacy both in monotherapy and in association with other drugs (particularly with molecules with direct antiproliferative effects) are urgently needed, as well as investigations into the role of sequential treatments.

Furthermore, additional research is necessary on how exposure to lenvatinib could change the biology of both the cancer and the patient's immune system. After a lot of skepticism, researchers are now convinced that the immune system plays a crucial role in controlling cancer cells and immune escape is probably one of the fundamental phenomena allowing the establishment and growth of tumors. Further studies on the interactions between small multi-targeted molecules and immune cells are urgently warranted.

Lenvatinib is a paradigmatic example of patients and clinicians moving toward to biological, orally administrable treatments with fewer upfront risks. However, many of these therapies are non-curative and should work as a "relay race" for subsequent treatments. Notably, there is still a lack of evidence on the best way to administer lenvatinib, in particular whether it should be used in combination or in sequence with other new and/or conventional treatments. The question of which drug or regimen to associate and in what sequence to administer them remains unknown and unexplored. Currently, 49 studies are ongoing to evaluate lenvatinib in monotherapy or in association with other small molecules, radiotherapy or immunotherapy. Among them, eight exploratory studies are evaluating lenvatinib in association with pembrolizumab in different neoplasms. Although interesting, a step back toward basic research should be taken when combining lenvatinib with immunotherapy, because no extensive studies have been conducted to elucidate the relations between lenvatinib exposure and immune system responses, nor has the selection of patients been clarified according to biological or immunological criteria. Eventual negative findings should take into account this interpretation, and further studies on the interactions between small multi-targeted molecules and immune cells are urgently needed.

Furthermore, from a clinical perspective, the increasing approval of drugs with a mechanism of action similar to lenvatinib should lead to the current response criteria being re-evaluated and modified. The overall tumor size may not necessarily reflect the tumor's response to this kind of therapy; other pathological and clinical parameters such as vessel microdensity and microenvironment, internal necrosis and/or "progression velocity", with accurate and standardized criteria, should be applied.

In conclusion, lenvatinib is an interesting and versatile option in anti-cancer treatment. However, additional improvements in clinical and translational studies, along with the support of innovative technologies, will result in a better selection of patients with the identification of genetic or specific pathway alterations predictive of efficacy or resistance, and in a better definition of efficacy criteria.

\section{Acknowledgments}

We thank the non-profit organization Lega Italiana Per La Lotta Contro i Tumori (LILT) of Naples, Dr Alessandra Trocino, Librarian at the Library of Istituto Nazionale Tumori Fondazione 'G Pascale', Naples, Italy, for her excellent bibliographic service and assistance, and Dr Stefania Santorufo for translation and correction of the manuscript.

\section{Disclosure}

The authors report no conflicts of interest in this work.

\section{References}

1. Tsimberidou AM. Targeted therapy in cancer. Cancer Chemother Pharmacol. 2015;76(6):1113-1132. doi:10.1007/s00280-015-2861-1

2. Scott LJ. Lenvatinib: first global approval. Drugs. 2015;75 (5):553-560. doi:10.1007/s40265-015-0383-0

3. Lenvima ${ }^{\circledR}$ (Lenvatinib). Summary of product characteristics. Hatfield (UK): Eisai Europe Limited, European Knowledge Centre, Mosquito Way; 2015.

4. Gupta A, Jarzab B, Capdevila J, Shumaker R, Hussein Z. Population pharmacokinetic analysis of lenvatinib in healthy subjects and patients with cancer. $B r \quad J$ Clin Pharmacol. 2016;81(6):1124-1133. doi:10.1111/bcp. 12907 
5. Dubbelman AC, Rosing H, Thijssen B, et al. Development and validation of LC-MS/MS assays for the quantification of E7080 and metabolites in various human biological matrices. J Chromatogr B Analyt Technol Biomed Life Sci. 2012;887-888:25-34. doi:10.1016/j.jchromb.2012.01.004

6. Koyama N, Saito K, Nishioka Y, et al. Pharmacodynamic change in plasma angiogenic proteins: a dose-escalation phase 1 study of the multi-kinase inhibitor lenvatinib. BMC Cancer. 2014;14:530. doi:10.1186/1471-2407-14-530

7. Tohyama O, Matsui J, Kodama K, et al. Antitumor activity of lenvatinib (e7080): an angiogenesis inhibitor that targets multiple receptor tyrosine kinases in preclinical human thyroid cancer models. J Thyroid Res. 2014;2014:638747. doi:10.1155/2014/638747

8. Matsui J, Yamamoto Y, Funahashi Y, et al. E7080, a novel inhibitor that targets multiple kinases, has potent antitumor activities against stem cell factor producing human small cell lung cancer H146, based on angiogenesis inhibition. Int J Cancer. 2008;122(3):664-671. doi:10.1002/ijc. 23131

9. Yamamoto Y, Matsui J, Matsushima T, et al. Lenvatinib, an angiogenesis inhibitor targeting VEGFR/FGFR, shows broad antitumor activity in human tumor xenograft models associated with microvessel density and pericyte coverage. Vasc Cell. 2014;6:6-18 doi:10.1186/2045-824X-6-6

10. Matsui J, Funahashi Y, Uenaka T, et al. Multi-Kinase inhibitor E7080 suppresses lymph node and lung metastases of human mammary breast tumor MDA-MB-231 via inhibition of vascular endothelial growth factor-receptor (VEGF-R) 2 and VEGF-R3 kinase. Clin Cancer Res. 2008;14(17):5459-5465. doi:10.1158/1078-0432.CCR07-5270

11. Ikuta K, Yano S, Trung VT, et al. E7080, a multi-tyrosine kinase inhibitor, suppresses the progression of malignant pleural mesothelioma with different proangiogenic cytokine production profiles. Clin Cancer Res. 2009;15(23):7229-7237. doi:10.1158/1078-0432.CCR$09-1980$

12. Ogino H, Hanibuchi M, Kakiuchi S, et al. E7080 suppresses hematogenous multiple organ metastases of lung cancer cells with nonmutated epidermal growth factor receptor. Mol Cancer Ther. 2011;10 (7):1218-1228. doi:10.1158/1535-7163.MCT-10-0707

13. Russo A, Franchina T, Ricciardi GRR, et al. Third generation EGFR TKIs in EGFR-mutated NSCLC: where are we now and where are we going. Crit Rev Oncol Hematol. 2017;117:38-47. doi:10.1016/j. critrevonc.2017.07.003

14. Bruheim S, Kristian A, Uenaka T, et al. Antitumour activity of oral E7080, a novel inhibitor of multiple tyrosine kinases, in human sarcoma xenografts. Int J Cancer. 2011;129:742-750. doi:10.1002/ijc.25922

15. Glen H, Mason S, Patel H, et al. E7080, a multi-targeted tyrosine kinase inhibitor suppresses tumor cell migration and invasion. $B M C$ Cancer. 2011;11:309. doi:10.1186/1471-2407-11-309

16. Wiegering A, Korb D, Thalheimer A, et al. E7080 (Lenvatinib), a multi-targeted tyrosine kinase inhibitor, demonstrates antitumor activities against colorectal cancer xenografts. Neoplasia. 2014;16 (11):972-981. doi:10.1016/j.neo.2014.09.008

17. Nakagawa T, Matsushima T, Kawano S, et al. Lenvatinib in combination with golvatinib overcomes hepatocyte growth factor pathway-induced resistance to vascular endothelial growth factor receptor inhibitor. Cancer Sci. 2014;105(6):723-730. doi:10.1111/cas.12409

18. Nakazawa Y, Kawano S, Matsui J, et al. Multitargeting strategy using Lenvatinib and golvatinib: maximizing anti-angiogenesis activity in a preclinical cancer model. Cancer Sci. 2015;106(2):201-207. doi:10.1111/cas.12581

19. Li J, Zou CL, Zhang ZM, et al. A multitargeted tyrosine kinase inhibitor Lenvatinib for the treatment of mice with advanced glioblastoma. Mol Med Rep. 2017;16(5):7105-7111. doi:10.3892/ mmr.2017.7456

20. Cabanillas ME, McFadden DG, Durante C. Thyroid cancer. Lancet. 2016;388(10061):2783-2795. doi:10.1016/S0140-6736(16)30172-6
21. Okamoto K, Kodama K, Takase K, et al. Antitumor activities of the targeted multi-tyrosine kinase inhibitor lenvatinib (E7080) against RET gene fusion-driven tumor models. Cancer Lett. 2013;340 (1):97-103. doi:10.1016/j.canlet.2013.07.007

22. Jing $\mathrm{C}$, Gao Z, Wang R, et al. Lenvatinib enhances the antitumor effects of paclitaxel in anaplastic thyroid cancer. Am J Cancer Res. 2017;7(4):903-912.

23. Ferrari SM, Bocci G, Di Desidero T, et al. Lenvatinib exhibits antineoplastic activity in anaplastic thyroid cancer in vitro and in vivo. Oncol Rep. 2018;39(5):2225-2234. doi:10.3892/or.2018.6306

24. Incio J, Tam J, Rahbari NN, et al. PlGF/VEGFR-1 signaling promotes macrophage polarization and accelerated tumor progression in obesity. Clin Cancer Res. 2016;22(12):2993-3004. doi:10.1158/ 1078-0432.CCR-15-1839

25. Rolny C, Mazzone M, Tugues S, et al. HRG inhibits tumor growth and metastasis by inducing macrophage polarization and vessel normalization through downregulation of PlGF. Cancer Cell. 2011;19 (1):31-44. doi:10.1016/j.ccr.2010.11.009

26. Lyden D, Hattori K, Dias S, et al. Impaired recruitment of bone-marrow-derived endothelial and hematopoietic precursor cells blocks tumor angiogenesis and growth. Nat Med. 2001;7 (11):1194-1201. doi:10.1038/nm1101-1194

27. Chen XW, Yu TJ, Zhang J, et al. CYP4A in tumor-associated macrophages promotes pre-metastatic niche formation and metastasis. Oncogene. 2017;36(35):5045-5057. doi:10.1038/onc.2017.118

28. Naoum GE, Morkos M, Kim B, Arafat W. Novel targeted therapies and immunotherapy for advanced thyroid cancers. Mol Cancer. 2018;17(1):51. doi:10.1186/s12943-018-0786-0

29. Mehnert JMVA, Brose M, Aggarwal R, et al. Pembrolizumab for advanced papillary or follicular thyroid cancer: preliminary results from the phase 1b KEYNOTE-028 study. In: American Society of Clinical oncology (ASCO) annual meeting: Chicago. J Clin Oncol. 2016;34(suppl; abstr 6091). doi:10.1200/JCO.2016.34.15_suppl.6091

30. Voron T, Colussi O, Marcheteau E, et al. VEGF-A modulates expression of inhibitory checkpoints on CD8+ T cells in tumors. J Exp Med. 2015;212(2):139-148. doi:10.1084/jem.20140559

31. Cai C, Tang J, Shen B, et al. Preclinical trial of the multi-targeted lenvatinib in combination with cellular immunotherapy ( $\mathrm{T}$ cells sensitized to neopalastic cells) for treatment of renal cell carcinoma. Exp Ther Med. 2017;14(4):3221-3228. doi:10.3892/ etm. 2017.4858

32. Taylor M, Dutcus CE, Schmidt E, et al. 776PD-A phase $1 \mathrm{~b}$ trial of lenvatinib (LEN) plus pembrolizumab (PEM) in patients with selected solid tumors. Ann Oncol. 2016;27(6):266-295. doi:10.1093/annonc/ mdw373.04

33. Yamada K, Yamamoto N, Yamada Y, et al. Phase I dose-escalation study and biomarker analysis of E7080 in patients with advanced solid tumors. Clin Cancer Res. 2011;15(8):2528-2537. doi:10.1158/ 1078-0432.CCR-10-2638

34. Garmy-Susini B, Varner JA. Circulating endothelial progenitor cells. Br J Cancer. 2005;93(8):855-858. doi:10.1038/sj.bjc.6602808

35. Mancuso P, Bertolini F. Circulating endothelial cells as biomarkers in clinical oncology. Microvasc Res. 2010;79(3):224-228. doi:10.1016/ j.mvr.2010.02.007

36. Boss DS, Glen H, Beijnen JH, et al. Phase I study of E7080, a multitargeted tyrosine kinase inhibitor, in patients with advanced solid tumours. $\mathrm{Br} \quad J$ Cancer. 2012;106(10):1598-1604. doi:10.1038/ bjc. 2012.154

37. Nishio M, Horai T, Horiike A, et al. Phase 1 study of Lenvatinib combined with carboplatin and paclitaxel in patients with non-small-cell lung cancer. Br J Cancer. 2013;109(3):538-544. doi:10.1038/bjc.2013.374

38. Molina AM, Hutson TE, Larkin J, et al. A phase $1 \mathrm{~b}$ clinical trial of the multi-targeted tyrosine kinase inhibitor lenvatinib (E7080) in combination with everolimus for treatment of metastatic renal cell carcinoma (RCC) cancer. Chemother Pharmacol. 2014;73 (1):181-189. doi:10.1007/s00280-013-2339-y 
39. Hong DS, Kurzrock R, Wheler JJ, et al. Phase I dose-escalation study of the multikinase inhibitor lenvatinib in patients with advanced solid tumors and in an expanded cohort of patients with melanoma. Clin Cancer Res. 2015;21(21):4801-4810. doi:10.1158/1078-0432.CCR-14-3063

40. Hong DS, Kurzrock R, Falchook GS, et al. Phase 1b study of lenvatinib (E7080) in combination with temozolomide for treatment of advanced melanoma. Oncotarget. 2015;6(40):43127-43134. doi:10.18632/ oncotarget.5756

41. Postow MA, Chesney J, Pavlick AC, et al. Nivolumab and ipilimumab versus ipilimumab in untreated melanoma. New Engl J Med. 2015;372:2006-2017. doi:10.1056/NEJMoa1414428

42. Motzer RJ, Hutson TE, Glen H, et al. Lenvatinib, everolimus, and the combination in patients with metastatic renal cell carcinoma: a randomised, phase 2, open-label, multicentre trial. Lancet Oncol. 2015;16(15):1473-1482. doi:10.1016/S1470-2045(15)00290-9

43. Schlumberger M, Jarzab B, Cabanillas ME, et al. A phase II trial of the multitargeted tyrosine kinase inhibitor lenvatinib (E7080) in advanced medullary thyroid cancer. Clin Cancer Res. 2016;22 (1):44-53. doi:10.1158/1078-0432.CCR-15-1127

44. Cabanillas ME, Schlumberger M, Jarzab B, et al. A phase 2 trial of lenvatinib (E7080) in advanced, progressive, radioiodine-refractory, differentiated thyroid cancer: a clinical outcomes and biomarker assessment. Cancer. 2015;121(16):2749-2756. doi:10.1002/cncr.29395

45. Schlumberger M, Tahara M, Wirth LJ, et al. Lenvatinib versus placebo in radioiodine-refractory thyroid cancer. $N$ Engl J Med. 2015;372(7):621-630. doi:10.1056/NEJMoa1406470

46. Ikeda K, Kudo M, Kawazoe S, et al. Phase 2 study of lenvatinib in patients with advanced hepatocellular carcinoma. J Gastroenterol. 2017;52(4):512-519. doi:10.1007/s00535-016-1263-4

47. Kudo M, Finn RS, Qin S, et al. Lenvatinib versus sorafenib in first-line treatment of patients with unresectable hepatocellular carcinoma: a randomised phase 3 non-inferiority trial. Lancet. 2018;391 (10126):1163-1173. doi:10.1016/S0140-6736(18)30207-1

48. Zhang Y, Weinberg RA. Epithelial-to-mesenchymal transition in cancer: complexity and opportunities. Front Med. 2018;12(4):361-373. doi:10.1007/s11684-018-0656-6
49. Lee YS, Kim SM, Kim BW, et al. Anti-cancer effects of HNHA and lenvatinib by the suppression of EMT-mediated drug resistance in cancer stem cells. Neoplasia. 2018;20(2):197-206. doi:10.1016/j. neo.2017.12.003

50. Lamouille S, Xu J, Derynck R. Molecular mechanisms of epithelial-mesenchymal transition. Nat Rev Mol Cell Biol. 2014;15 (3):178-196. doi:10.1038/nrm3758

51. Al-Abd AM, Alamoudi AJ, Abdel-Naim AB, Neamatallah TA, Ashour OM. Anti-angiogenic agents for the treatment of solid tumors: potential pathways, therapy and current strategies - a review. J Adv Res. 2017;8(6):591-605. doi:10.1016/j.jare.2017.06.006

52. Rexer BN, Engelman JA, Arteaga CL. Overcoming resistance to tyrosine kinase inhibitors: lessons learned from cancer cells treated with EGFR antagonists. Cell Cycle. 2009;8(1):18-22. doi:10.4161/ cc.8.1.7324

53. Sennino B, Md DM. Controlling escape from angiogenesis inhibitors. Nat Rev Cancer. 2012;12(10):699-709. doi:10.1038/nrc3366

54. Shojaei F, Lee JH, Simmons BH, et al. HGF/c-Met acts as an alternative angiogenic pathway in sunitinib-resistant tumors. Cancer Res. 2010;70:10090-10100. doi:10.1158/0008-5472.CAN-10-0489

55. Luo M, Fu LW. Redundant kinase activation and resistance of EGFR-tyrosine kinase inhibitors. Am J Cancer Res. 2014;4 (6):608-628.

56. Saji M, Ringel MD. The PI3K-AKT-mTOR pathway in initiation and progression of thyroid tumors. Mol Cell Endocrinol. 2010;321 (1):20-28. doi:10.1016/j.mce.2009.10.016

57. Ghidini M, Petrelli F, Ghidini A, et al. Clinical development of mTor inhibitors for renal cancer. Expert Opin Investig Drugs. 2017;26(11):1229-1237. doi:10.1080/13543784.2017.1384813

58. Scoazec JY. Angiogenesis in neuroendocrine tumors: therapeutic applications. Neuroendocrinology. 2013;97(1):45-56. doi:10.1159/ 000338371

59. Capozzi M, Von Arx C, De Divitiis C, et al. Antiangiogenic therapy in pancreatic neuroendocrine tumors. Anticancer Res. 2016;36 (10):5025-5030. doi:10.21873/anticanres.11071

\section{Publish your work in this journal}

Cancer Management and Research is an international, peer-reviewed open access journal focusing on cancer research and the optimal use of preventative and integrated treatment interventions to achieve improved outcomes, enhanced survival and quality of life for the cancer patient.
The manuscript management system is completely online and includes a very quick and fair peer-review system, which is all easy to use. Visit http://www.dovepress.com/testimonials.php to read real quotes from published authors. 